\title{
Diffraction from a wavelet point of view
}

\author{
Levent Onural \\ Department of Electrical and Electronics Engineering, Bilkent University, Bilkent, TR-06533 Ankara, Turkey
}

Received December 22, 1992

\begin{abstract}
The system impulse response representing the Fresnel diffraction is shown to form a wavelet family of functions. The scale parameter of the wavelet family represents the depth (distance). This observation relates the diffraction-holography-related studies and the wavelet theory. The results may be used in various optical applications such as designing robust volume optical elements for optical signal processing and finding new formulations for optical inverse problems. The results also extend the wavelet concept to the nonbandpass family of functions with the implication of new applications in signal processing. The presented wavelet structure, for example, is a tool for space-depth analysis.
\end{abstract}

The wavelet concept and its applications have been shown to be useful in various signal-processing problems (see, for example, Refs. 1 and 2). Recently there have been various studies that implement wavelet analysis by using optical processing techniques (see, for example, Ref. 3 and 4). However, it appears that the fundamental relationship between the essentials of optics and the wavelet concept has gone undetected: the propagation of waves itself carries the wavelet characteristics, inherently. Although this observation is trivial, its implications may pave the road for various novel applications in optics as well as in signal processing.

The wavelet theory has been well developed over the past decade, so various results can be immediately transferred to optics and optical applications. For example, holography can be revisited with a new interpretation and applications; there could be distributed optical elements to achieve desired processes in larger volumes compared with rather thin lenses and masks, and new tools for inverse problems to find the sources from distant observations may be rather easily developed. One can use the presented wavelet analysis to determine the location of particles in three-dimensional (3-D) space from their holograms. The search of the unknown depth of a particle at a specific location of the two-dimensional (2-D) observation window is analogous to the search for the unknown dominant frequency at a specific location of a 2-D signal by using conventional wavelet analysis. Forming frames ${ }^{1}$ by discretizing the depth or even finding an orthonormal complete basis (for example, to be used in discrete depth volume holography) can be immediately achieved by carrying the results of existing wavelet analysis.

The wavelet family discussed here also extends the wavelet concept and its applications in signal processing. Conventionally, the wavelets are used for time (space)-frequency analysis of signals. This requirement is consistent with the admissibility condition as derived in Ref. 1, which prohibits wavelets that are not bandpass functions. However, the presented family of wavelets is not band limited, and for that reason it is not suitable for time (space)-frequency analysis. A new admissibility condition that extends the one given in Ref. 1 is already proved, so that wavelets as presented in this Letter can also be used for forward and inverse wavelet transform. This wavelet family is used for space-depth analysis, where the scale parameter no longer represents a shift in the covered band but rather is a distance (depth) parameter.

The principles of diffraction can be found in classical references such as Born and Wolf. ${ }^{5}$ In 3-D space, the field at a distance $z$ from a planar distribution $\psi(x, y)$ is given by the Huygens-Fresnel approximation as $^{6}$

$$
\begin{aligned}
\psi_{z}(x, y)= & \frac{1}{j \lambda z} \exp \left(j \frac{2 \pi}{\lambda} z\right) \int_{\xi} \int_{\eta} \psi(\xi, \eta) \\
& \times \exp \left\{j \frac{\pi}{\lambda z}\left[(x-\xi)^{2}+(y-\eta)^{2}\right]\right\} \mathrm{d} \xi \mathrm{d} \eta,
\end{aligned}
$$

where $\lambda$ is the wavelength. This relationship may be represented as a linear shift-invariant system with an impulse response, ${ }^{7}$

$$
h_{z}(x, y)=\frac{1}{j \lambda z} \exp \left(j \frac{2 \pi}{\lambda} z\right) \exp \left[j \frac{\pi}{\lambda z}\left(x^{2}+y^{2}\right)\right]
$$

which yields

$$
\psi_{z}(x, y)=\psi(x, y) * * h_{z}(x, y),
$$

where $* *$ represents the 2 -D convolution.

A similar representation may also be given for waves propagating in 2-D space, where both the object distribution and the diffraction pattern are one dimensional. This case is omitted here.

With these observations, it is easy to see that the convolution kernel of Eq. (3) forms a continuous wavelet family for 2-D function space. Classically, the wavelets are a family of functions, obtained from one single function, indexed by two labels as ${ }^{1}$

$$
w^{(a, b)}=|a|^{-1 / 2} w\left(\frac{x-b}{a}\right),
$$

where $w$ is a function that satisfies

$$
C_{w}=\int_{\omega}|\omega|^{-1}|W(\omega)|^{2} \mathrm{~d} \omega<\infty .
$$


$W(\omega)$ is the Fourier transform of $w(x)$. The inner product of a function $\phi$ with one of the wavelet functions gives a coefficient corresponding to the parameters $a$ and $b$. The set of such inner products for all $a$ 's and $b$ 's constitutes a representation for the function $\phi$ as

$$
\phi=C_{w}{ }^{-1} \iint\left\langle w^{(a, b)}, \phi\right\rangle w(a, b) \frac{\mathrm{d} a}{a^{2}} \mathrm{~d} b,
$$

where the inner product is defined as

$$
\langle f, g\rangle=\int \overline{f(x)} g(x) \mathrm{d} x .
$$

The extension to higher dimensions is straightforward. In our case, the extension to the 2-D space is achieved as follows. The family of functions is obtained from a single function by use of three parameters:

$$
w^{(a, b, c)}=K_{a} w\left(\frac{x-b}{a}, \frac{y-c}{a}\right) .
$$

It is easy to see from Eqs. (2) and (8) that the diffraction kernel indeed has the shift and the scaling properties associated with the wavelet family. This is shown by defining the generic wavelet as

$$
h(x, y)=\exp \left[j\left(x^{2}+y^{2}\right)\right]
$$

and obtaining the family of wavelets from this function:

$$
h^{(a, b, c)}=K_{a} h\left(\frac{x-b}{a}, \frac{y-c}{a}\right) .
$$

Here $a=\sqrt{\lambda z / \pi}, b=\xi$, and $c=\eta$, and the amplitude constant is given by

$$
K_{a}=\frac{\exp \left(j \frac{2 \pi}{\lambda} z\right)}{j \lambda z}=\exp \left\{j\left[2\left(\frac{\pi a}{\lambda}\right)^{2}-\frac{\pi}{2}\right]\right\} \frac{1}{\pi a^{2}} .
$$

Again, the amplitude scaling and the integrability conditions are not like the conditions given by relations (4) and (5), but similar conditions for these wavelet functions exist.

As is shown by Eq. (3), the diffraction can be written as a convolution. Furthermore, it can be also be shown that the translated kernels with the same scale variable $a$ form a complete orthogonal set. This implies that if the projections of a function $\phi(x, y)$ onto functions $h^{(a, b, c)}$ for all $b$ and $c$ but for a constant (nonzero) $a$ are known, then the function can be uniquely recovered from these projections. This is, of course, a well-known result in diffraction: One may start from a field distribution $\psi(x, y)$ and get the diffracted field over a plane at a distance $z$ by using Eq. (1). If desired, the original distribution can be obtained from the diffracted field by using backpropagation as

$$
\begin{aligned}
& \psi(x, y)=\psi_{z}(x, y) * * h_{z}^{*}(x, y) \\
& \psi(x, y)=\frac{1}{-j \lambda z} \exp \left(-j \frac{2 \pi}{\lambda} z\right) \int_{\xi} \int_{\eta} \psi_{z}(\xi, \eta) \\
& \quad \times \exp \left\{-j \frac{\pi}{\lambda z}\left[(x-\xi)^{2}+(y-\eta)^{2}\right]\right\} \mathrm{d} \xi \mathrm{d} \eta
\end{aligned}
$$

Since $h_{z}(x, y) * * h_{z}{ }^{*}(x, y)=\delta(x, y), \psi(x, y) * *$ $h_{z}(x, y) * * h_{z}{ }^{*}(x, y)=\psi(x, y){ }^{7}$ Note that $h_{z}{ }^{*}=$ $h_{-z}$, as expected: one represents the propagation of the wave in the positive direction, while the other one represents the propagation in the negative direction.

Now let us consider the 3-D field generated by a 2-D diffracting obstacle. Since Eq. (1) is valid for any $z$ that satisfies the Fresnel approximation, we may find the 3-D field as $\psi(x, y, z)=\psi_{z}(x, y)$. Since the 2-D original $\psi(x, y)$ can be reconstructed from $\psi_{z}(x, y)$ for a fixed arbitrary $z$, it can also be reconstructed from $\psi(x, y, z)$, where $z$ has a variation. In this case the reconstruction is

$$
\begin{gathered}
\psi(x, y)=\frac{1}{C} \int_{z} \frac{1}{-j \lambda z} \exp \left(-j \frac{2 \pi}{\lambda} z\right) \int_{\xi} \int_{\eta} \psi(\xi, \eta, z) \\
\times \exp \left\{-j \frac{\pi}{\lambda z}\left[(x-\xi)^{2}+(y-\eta)^{2}\right]\right\} \mathrm{d} \xi \mathrm{d} \eta \mathrm{d} z,
\end{gathered}
$$

where the integration with respect to $z$ is over a finite domain that does not include the zone that violates the Fresnel approximation. The value of $C$ depends on the limits of integration with respect to $z$. Therefore the diffraction process, i.e., the 3-D field from a 2-D obstacle, can be seen as the wavelet transform. The reconstruction as given by Eq. (13) is the inverse wavelet transform. This is a new look from the framework of wavelets to a well-known result.

The volume diffraction pattern is, of course, redundant. The possible use of this redundancy is for distributed filtering: the function of the filter will be quite insensitive to errors associated with the design or the implementation as a consequence of the redundancy. The filter, in this case, is the operation corresponding to (i) taking the wavelet transform, (ii) multiplying the wavelet coefficients by filter parameters, and (iii) taking the inverse wavelet transform. All these operations can be translated to the diffraction case as (i) obtaining a volume diffraction pattern by illuminating a diffracting pattern, (ii) building a volume mask (which, of course, does not block out the light significantly) and placing it into the volume of diffracted beam, and (iii) inverting the diffraction (maybe by a lens) as in conventional optical filtering. Here the volume mask should not be too obstructive. That is, the mask should consist of dustlike opaque elements with low concentration, and/or it should consist of refractive-index variations.

Similar interpretation may be carried to holography as well. In-line holography is conceptually similar to diffraction; the characterizing difference is the magnitude operating at the imaging plane:

$$
I_{z}(x, y)=\left|\psi(x, y) * * h_{z}(x, y)\right|^{2},
$$


where $I_{z}$ is the in-line hologram. Usually an object distribution $s(x, y)$ is introduced such that the field emerging from the diffracting (object) plane when it is illuminated by a reference plane wave is $1-s(x, y)=$ $\psi(x, y) .6,7$ It is required that $\|s\| \ll 1$. In that case, the hologram can be written as

$$
\left.I_{z}(x, y)=\mid(1-s) * * h_{z}\right)\left.\right|^{2} \approx 1-s * * h_{z}-s^{*} * * h_{z}^{*} .
$$

If the object distribution takes only real values, it is possible to write the in-line hologram approximately as ${ }^{7}$

$$
I_{z}(x, y)=1-s(x, y) * * 2 \operatorname{Re}\left\{h_{z}(x, y)\right\} .
$$

Defining $2 \operatorname{Re}\left\{h_{z}\right\}=g_{z}(x, y)$, one can write the hologram as

$$
I_{z}(x, y)=1-s * g_{z}
$$

Note that

$$
g_{z}(x, y)=\frac{2}{\lambda z} \sin \left[\frac{\pi}{\lambda z}\left(x^{2}+y^{2}\right)\right] .
$$

The family of functions can be obtained from a single generic function, $g(x, y)=\sin \left(x^{2}+y^{2}\right)$, as

$$
g^{(a, b, c)}(x, y)=K_{a g}\left(\frac{x-b}{a}, \frac{y-c}{a}\right) .
$$

Here $a=\sqrt{\lambda z / \pi}$, as in diffraction. This $a$ is the depth (scale) parameter. Again, as before, $b$ and $c$ are the shift parameters. The amplitude scale constant $K_{a}$ is defined in this case as

$$
K_{a}=\frac{2}{\lambda z}=\frac{2}{\pi a^{2}}
$$

An interesting observation is that the set of functions for a constant depth parameter $a$ does not constitute a complete basis in this case, because $G^{(a, 0,0)}(u, v)=\mathcal{F}\left\{g^{(a, 0,0)}\right\}=2 \cos \left[a^{2} / 4\left(u^{2}+v^{2}\right)\right]$, and therefore the inverse transformation does not exist since $G^{(a, 0,0)}(u, v)$ has zeros. ${ }^{7}$

However, it is interesting to note that, as a consequence of the wavelet inversion, the original object field can be recovered by using a volume hologram, as expected. Thus a well-known result in holography is again shown by using the wavelet analysis. However, from the wavelet theory it is known that the basis obtained by continuously changing the parameter $a$ is highly redundant. This redundancy can be reduced (even eliminated) by discretizing the depth parameter $a$ properly.
It is shown that fundamental optical phenomena such as diffraction and holography can be looked at from a wavelet framework. Essentially, one can put any form of wave propagation into a wavelet framework by following similar steps. As a consequence, there is a vast possibility of applications that readily follow various results of wavelet theory. The wavelet theory results in eliminating the redundancy by introducing discrete parameter spaces as in forming frames, and orthonormal basis may be utilized to convert many optical volume processes to multiplanar operations. One possibility is multiplane holography. The same idea may be useful also in various wave-propagation-related areas such as antenna design and imaging using intruding waves. On the other hand, the same theory may be used in the other direction to use the inherent redundancy for improving the noise immunity of the desired operation. Distributed filtering over a volume may be appealing, for example, in the design of optical processors distributed over an optical fiber.

The analysis of a hologram by using digital techniques to extract object related information may find a useful theoretical framework as a consequence of the presented wavelet diffraction relation. ${ }^{8}$

Another possibility is to provide a new framework to study different wave-propagation media. For example, wave propagation in graded-index media with special radial refractive-index distribution ${ }^{9}$ can be put into a wavelet framework, yielding new ideas both in analysis and synthesis.

The diffraction and related ideas and their wavelet nature are also interesting for the sake of signal processing itself. New signal representations and analysis tools may emerge based on the diffraction principles.

\section{References}

1. I. Daubechies, IEEE Trans. Inf. Theory 36, 961 (1990).

2. S. Mallat, IEEE Trans. Pattern Anal. Mach. Intell. 31, 674 (1989).

3. Y. Sheng, D. Roberge, and H. H. Szu, Opt. Eng. 31, 1840 (1992).

4. Y. Li and Y. Zhang, Opt. Eng. 31, 1865 (1992).

5. M. Born and E. Wolf, Principles of Optics, 5th ed. (Pergamon, London, 1975), Chap. 8, pp. 370-387.

6. G. A. Tyler and B. J. Thompson, Opt. Acta 23, 685 (1976).

7. L. Onural and P. D. Scott, Opt. Eng. 26, 1124 (1987).

8. L. Onural and M. T. Özgen, J. Opt. Soc. Am. A 9, 252 (1992).

9. D. Mendlovic and H. M. Ozaktas, "Fractional Fourier transforms and their optical implementation. Part I," J. Opt. Soc. Am. A (to be published). 